RESEARCH ARTICLE

\title{
$202 \mathrm{~W}$ dual-end-pumped Tm:YLF laser with a VBG as an output coupler
}

\author{
Disheng Wei, Shuyi Mi, Ke Yang, Junhui Li, Jinwen Tang, Baoquan Yao, Tongyu Dai, and \\ Xiaoming Duan \\ National Key Laboratory of Tunable Laser Technology, Harbin Institute of Technology, Harbin 150001, China \\ (Received 6 July 2021; revised 9 August 2021; accepted 19 August 2021)
}

\begin{abstract}
We demonstrated a $202 \mathrm{~W}$ Tm:YLF slab laser using a reflecting volume Bragg grating (VBG) as an output coupler at room temperature. Two kinds of active heat dissipation methods were used for the VBG to suppress the shift of wavelength caused by its increasing temperature. The maximum continuous wave (CW) output power of $202 \mathrm{~W}$ using the microchannel cooling was obtained under the total incident pump power of $553 \mathrm{~W}$, the corresponding slope efficiency and optical-to-optical conversion efficiency were $39.7 \%$ and $36.5 \%$, respectively. The central wavelength was $1908.5 \mathrm{~nm}$ with the linewidth (full width at half maximum) of $0.57 \mathrm{~nm}$. Meanwhile, with the laser output increasing from 30 to $202 \mathrm{~W}$, the total shift was about $1.0 \mathrm{~nm}$, and the wavelength was limited to two water absorption lines near $1908 \mathrm{~nm}$. The beam quality factors $M^{2}$ were measured to be 2.3 and 4.0 in $x$ and $y$ directions at $202 \mathrm{~W}$.
\end{abstract}

Keywords: hundred watt level; reflecting volume Bragg grating; Tm:YLF laser

\section{Introduction}

Lasers with $2 \mu \mathrm{m}$ waveband have been widely used in various technical fields, including medical laser application $^{[1]}$, remote sensing ${ }^{[2,3]}$, and 3-5 $\mu \mathrm{m}$ nonlinear frequency conversion as the pump source ${ }^{[4]}$, owing to their excellent characteristics of being located in the atmospheric window, strong absorption band of water, and safety zone of human eyes $^{[4-6]}$. At room temperature, a laser crystal doped with trivalent rare earth holmium (Ho) ions has a long upper-state lifetime and a large emission cross section, and thus is an efficient $2 \mu \mathrm{m}$ solid laser material. As excellent pumping sources for Ho lasers, thulium-doped materials have several attractive properties of light generation, including a wide emission bandwidth, long lifetime upper laser level, and high quantum efficiency potential owing to the two-to-one cross-relaxation process ${ }^{[7,8]}$. As far as Tm:YLF material is concerned, it has a strong absorption near $791 \mathrm{~nm}^{[9]}$, which is suitable for laser diode (LD) pumping. The cross section at $1908 \mathrm{~nm}$ in the direction of $\sigma$ polarization is the largest ${ }^{[9]}$, which exactly overlaps with the absorption spectrum of

Correspondence to: B. Yao, National Key Laboratory of Tunable Laser Technology, Harbin Institute of Technology, Harbin 150001, China. Email: yaobq08@hit.edu.cn
Ho:YAG ${ }^{[7]}$. However, the absorption spectrum of Ho:YAG is relatively narrow ${ }^{[10]}$, and there are a lot of $\mathrm{H}_{2} \mathrm{O}$ absorption lines in the near-infrared spectral region ${ }^{[11]}$. Therefore, it is necessary to limit the output linewidth of Tm:YLF laser.

A reflecting volume Bragg grating (VBG) is a kind of narrow-band-pass filter element based on the Bragg condition. It can be used to replace the laser resonator mirror of Tm:YLF laser to achieve narrow-linewidth laser output ${ }^{[12-14]}$. However, the minute absorption of VBG as a cavity end mirror can cause some adverse effects, including the change of spatial period and refractive index, the decrease of diffraction efficiency, and surface deformation, which will cause changes in laser performance ${ }^{[15,16]}$, especially the obvious wavelength shift. Owing to the existence of mass water absorption lines in the near-infrared spectral region ${ }^{[11]}$, the shift is very unfriendly for Tm:YLF laser, especially at the output power level of hundreds of watts. From this point of view, effective heat dissipation for VBG is recommended to prevent its performance degradation in this experiment.

At present, there are few reports about Tm:YLF lasers with an output power level of $200 \mathrm{~W}$, but the previous reports are based on Tm crystals dual-end-pumped by LD stacks, where the beam quality factor $M^{2}$ in one direction is very poor. In 2013, Li et al. reported that a $200 \mathrm{~W}$ Innoslab 


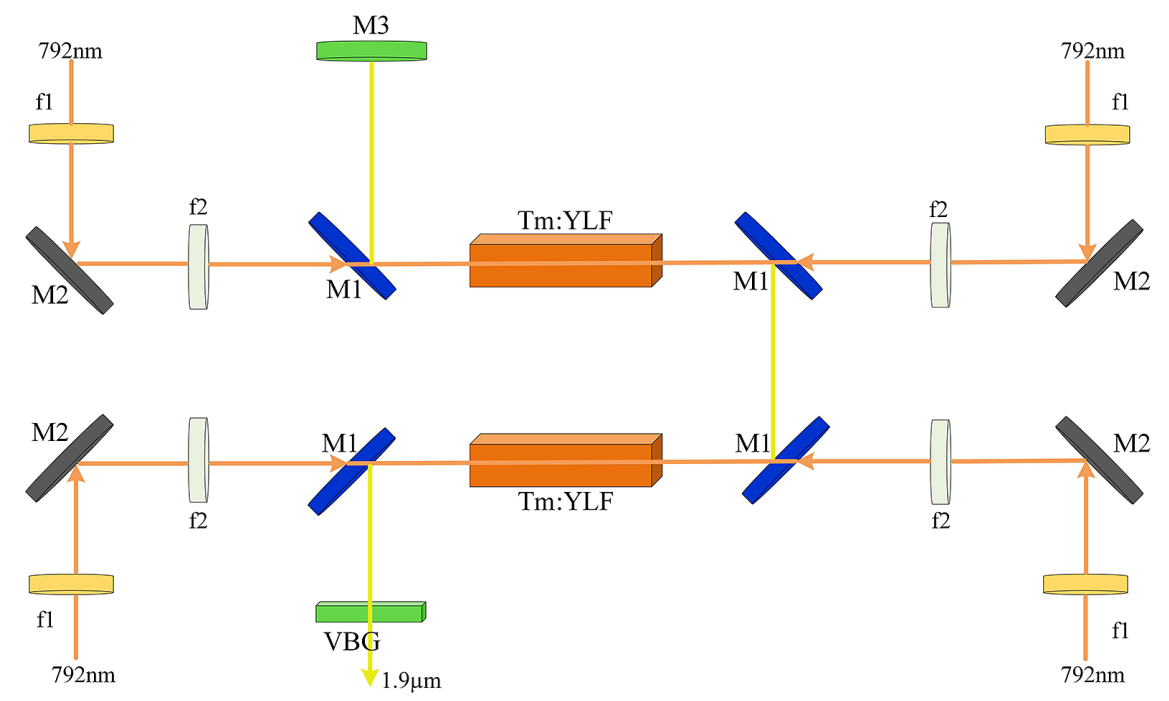

Figure 1. Diagrammatic sketch of the experimental setup.

Tm:YLF laser output had a strong elliptical beam shape with a Gaussian distribution along the semi-minor axis and a top-hat-like distribution beam shape along the semi-major axis $^{[17]}$. In 2019, Mao and Wang reported a $213 \mathrm{~W}$ Tm:YLF Innoslab wavelength-selected laser, and the corresponding beam quality factors were $M^{2}{ }_{x}=520$ and $M_{y}{ }_{y}=2.1$ at only $120 \mathrm{~W}^{[18]}$. Based on the previous reports, the beam quality is very poor in one direction and difficult to control, which has badly limited its application.

In this paper, a $202 \mathrm{~W}$ Tm:YLF laser with relatively good beam quality and narrow linewidth near $1908 \mathrm{~nm}$ was demonstrated by using two crystals in series and a dual-endpumped structure for each crystal. To the best of the authors' knowledge, this is the first report on such high-power output with VBG as an output coupler for a Tm:YLF slab laser. To suppress the wavelength shift to the water absorption line near $1908.9 \mathrm{~nm}$, the active heat dissipation methods were used to cool the VBG, including microchannel cooling and temperature control with a thermoelectric cooler (TEC). The results show that active cooling can effectively restrain the growth rate of the laser output wavelength. Without active cooling, the laser wavelength was $1908.8 \mathrm{~nm}$ at $160 \mathrm{~W}$; with TEC, the laser wavelength was $1908.64 \mathrm{~nm}$ at $201 \mathrm{~W}$; with microchannel cooling, the laser wavelength was $1908.5 \mathrm{~nm}$ at $202 \mathrm{~W}$. In this way, the Tm:YLF laser was operated at $1908.5 \mathrm{~nm}$ with linewidth (full width at half maximum, FWHM) $0.57 \mathrm{~nm}$. Under an incident pump power of $553 \mathrm{~W}$, the maximum continuous wave (CW) output power of $202 \mathrm{~W}$ at $1908.5 \mathrm{~nm}$ was obtained, corresponding to the slope efficiency of $39.7 \%$ and optical-to-optical conversion efficiency of $36.5 \%$, where the beam quality factors $M^{2}$ were 2.3 and 4.0 for horizontal and vertical directions, respectively.

\section{Experimental setup}

The experimental setup of the Tm:YLF slab laser is shown in Figure 1. A double-crystal series and a dual-end-pump configuration were utilized in this experiment. The four pump lasers were $150 \mathrm{~W}$ fiber-coupled LDs with core diameter of $400 \mu \mathrm{m}$, numerical aperture of 0.2 , and central wavelength of $792 \mathrm{~nm}$ at the highest power. The radiation of each LD was coupled into the laser crystal by the same focusing optical system composed of two spherical lenses with focal lengths $f_{1}=13 \mathrm{~mm}$ and $f_{2}=55 \mathrm{~mm}$, generating a pump beam diameter of about $1.7 \mathrm{~mm}$ within the laser crystals. Between $f_{1}$ and $f_{2}$, a $45^{\circ}$ high-reflection (HR) mirror M2 was used to change the direction of the $792 \mathrm{~nm}$ radiation. In this work, two 2 at. $\%, 2 \mathrm{~mm} \times 6 \mathrm{~mm} \times 40 \mathrm{~mm}$ and $a$-cut Tm:YLF crystals were wrapped with indium foil and mounted in copper blocks cooled by water at a temperature of $290 \mathrm{~K}$. The two crystals were connected in series in the resonant cavity. The whole cavity consisted of four $45^{\circ}$ dichroic mirrors M1, a VBG, and a reflector M3. One side of the mirror M1 was coated with $792 \mathrm{~nm}$ anti-reflection coating, the other side was coated with HR material at 1908$2090 \mathrm{~nm}(R>97 \%)$ and highly transmitting at $792 \mathrm{~nm}$. The mirror M3 was a concave mirror with a radius of curvature of $200 \mathrm{~mm}$ and $\mathrm{HR}$ coated at $1.9 \mu \mathrm{m}(R>99.7 \%)$. As the output coupler of the laser, the VBG had a clear aperture of $3 \mathrm{~mm} \times 4 \mathrm{~mm}$ and a thickness of $2.4 \mathrm{~mm}$ and its diffraction efficiency was about $58.4 \%$ at $1907.3 \mathrm{~nm}$, whose spectral selectivity was less than $0.7 \mathrm{~nm}$. It was wrapped in indium foil and mounted on a copper heat sink. In addition, the physical cavity length of the Tm:YLF laser was $230 \mathrm{~mm}$. 


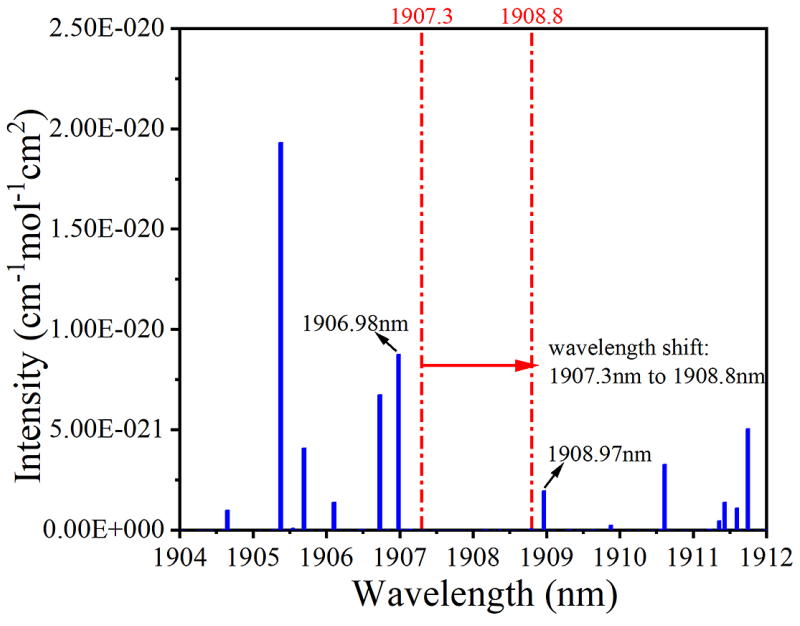

Figure 2. Water absorption spectrum near $1908 \mathrm{~nm}$ (plotted using HITRAN data ${ }^{[11]}$ ) and laser wavelength shift.

\section{Results and discussion}

First, without active cooling, the emission wavelength shifted from 1907.3 to $1908.8 \mathrm{~nm}$ and the corresponding volume grating temperature increased from 302 to $391 \mathrm{~K}$, when the output power was increased from 7 to $160 \mathrm{~W}$, as shown in Figure 3(a). The temperature was measured three times and averaged at the same output power, recorded by a temperature-measuring infrared thermal imager (DALI LT7P). The wavelength was shifted $1.5 \mathrm{~nm}$. This meant that if we continued to increase the output power of the laser, its wavelength was likely to drift to the water absorption line near $1908.97 \mathrm{~nm}$ as shown in Figure 2, which would cause damage to the laser. The reason for the wavelength shift is that VBG has a small absorption coefficient (less than $0.01 \mathrm{~cm}^{-1[19]}$ ) for $1.9 \mu \mathrm{m}$ laser, and the absorbed energy is converted into heat, which causes a temperature rise and distortion especially in high-flux laser irradiation.

Owing to the change in spatial period caused by the nonuniform thermal expansion and the change in refractive index caused by the thermal dispersion coefficient, some wavelength components of the incident laser deviate from the Bragg condition,

$$
\lambda_{\mathrm{B}}=2 n_{0} \Lambda \sin \theta_{\mathrm{B}},
$$

where $\lambda_{\mathrm{B}}$ is the Bragg wavelength, $n_{0}$ is the refractive index, and $\Lambda$ is the grating period ${ }^{[20]}$. The influence of temperature change on Bragg wavelength can be analyzed by the derivative of the Bragg condition with respect to temperature $T$ at normal incidence,

$$
\frac{\mathrm{d} \lambda_{\mathrm{B}}}{\mathrm{d} T}=2 \frac{\mathrm{d} n_{0}}{\mathrm{~d} T} \Lambda+2 \frac{\mathrm{d} \Lambda}{\mathrm{d} T} n_{0}=\lambda_{\mathrm{B}} \cdot\left(\frac{1}{n} \frac{\mathrm{d} n_{0}}{\mathrm{~d} T}+\rho\right) .
$$

In Equation (2), $\rho$ is the thermal expansion coefficient and $\mathrm{d} n_{0} / \mathrm{d} T$ is the thermal dispersion coefficient. For PTR glass, the values are $\rho=8.5 \times 10^{-6} \mathrm{~K}^{-1[19]}$ and $\mathrm{d} n_{0} / \mathrm{d} T=$ $5 \times 10^{-8} \mathrm{~K}^{-1[21]}$. As the thermal dispersion coefficient is two orders of magnitude smaller than the thermal expansion coefficient, the influence of thermal dispersion coefficient can be ignored. Hence, Equation (2) can be further simplified as follows:

$$
\frac{\mathrm{d} \lambda_{\mathrm{B}}}{\mathrm{d} T}=\lambda_{\mathrm{B}} \cdot \rho .
$$

The general solution of Equation (3) is

$$
\lambda_{\mathrm{B}}=A_{\lambda_{\mathrm{B} 0}} \cdot e^{\rho T} .
$$

Here $A_{\lambda_{\mathrm{B} 0}}$ is a constant, which depends on a VBG with a given Bragg wavelength. As $\rho$ is very small, Equation (4) can be approximated by the first-order McLaughlin approximation,

$$
\lambda_{\mathrm{B}}=A_{\lambda_{\mathrm{B} 0}}+A_{\lambda_{\mathrm{B} 0}} \rho T .
$$

Equation (5) shows that the dependence of Bragg wavelength on temperature can be approximately linear, described by the slope of the expression with $\mathrm{d} \lambda_{\mathrm{B}} / \mathrm{d} T$. We assume that the Bragg wavelength of the VBG is $1907.3 \mathrm{~nm}$ at $293 \mathrm{~K}$ to calculate the dependence. The constant $A_{\lambda_{\mathrm{B} 0}}$ is about $1902.55 \mathrm{~nm}$ and $\mathrm{d} \lambda_{\mathrm{B}} / \mathrm{d} T$ is roughly $0.0162 \mathrm{~nm} \cdot \mathrm{K}^{-1}$, which is close to the actual values of $1902.98 \mathrm{~nm}$ and $0.0147 \mathrm{~nm} \cdot \mathrm{K}^{-1}$ acquired by fitting the experimental data, as shown in Figure 3(b). We can also calculate the wavelength shift of $1.44 \mathrm{~nm}$ from 302 to $391 \mathrm{~K}$, which is basically consistent with the experimental value $1.50 \mathrm{~nm}$. Therefore, in order to prevent water absorption damage to the laser, effective measures must be taken to restrain the laser wavelength shift caused by the increase of VBG temperature.

The output characteristics with different heat dissipation methods of the VBG are compared in Figure 4. There were two active cooling methods, the first was a microchannel cooler and the second was a TEC. The ambient temperature of the VBG was controlled at $290 \mathrm{~K}$ with these two methods. As shown in Figure 4(a), the slope efficiencies under different conditions were $39.7 \%, 38.2 \%$, and $39.9 \%$ and the corresponding thresholds were 54.7, 51.5, and 57.6 $\mathrm{W}$, respectively. According to the existing literature, it can be shown that a VBG used as a cavity end mirror of laser has the thermal lensing effect ${ }^{[22]}$ and causes reduction of diffraction efficiency ${ }^{[15,16,20]}$, which leads to the change of the mode volume and the reflectivity of the VBG, thus changing the output characteristics of the laser. Hence, after the active cooling, the light output threshold decreased slightly, and the efficiency had a slight change. In Figure 4(a), it should be noted that the total incident pump power was different 

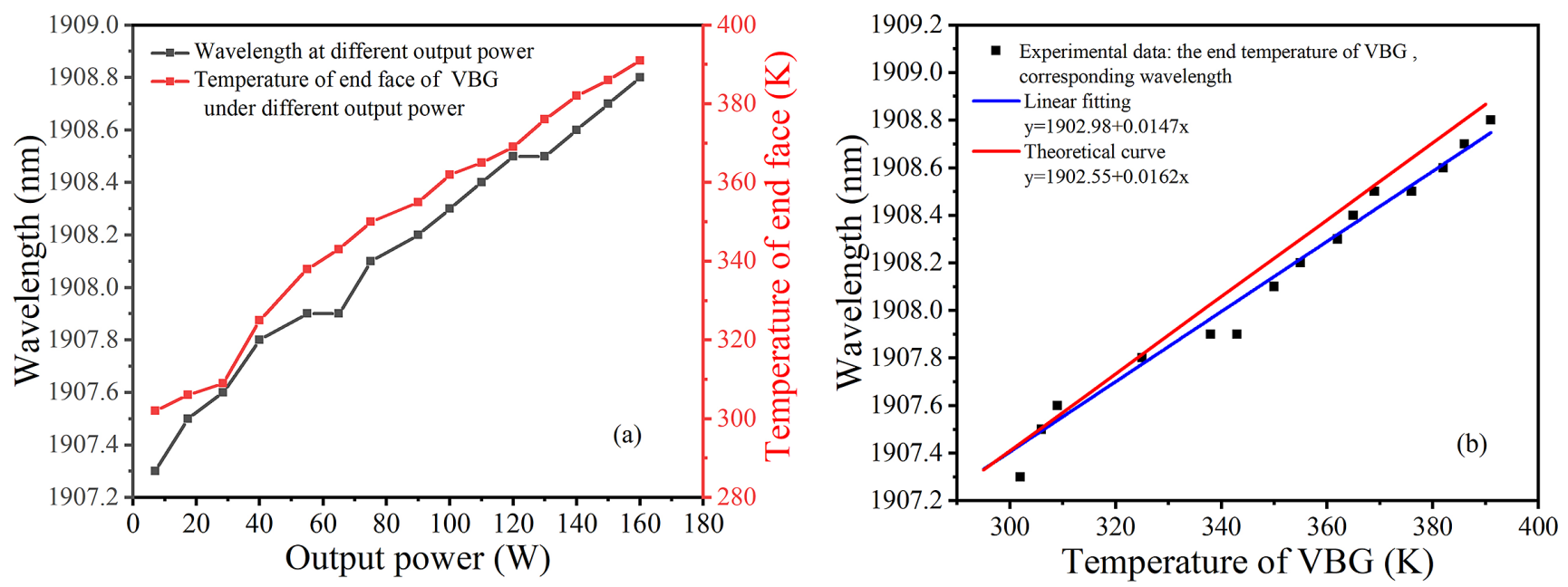

Figure 3. Dependence of the laser wavelength on temperature without active cooling in the VBG: (a) wavelength at different output power and corresponding temperature under different output power; (b) fitting of the relationship between wavelength and temperature, and corresponding theoretical curve.
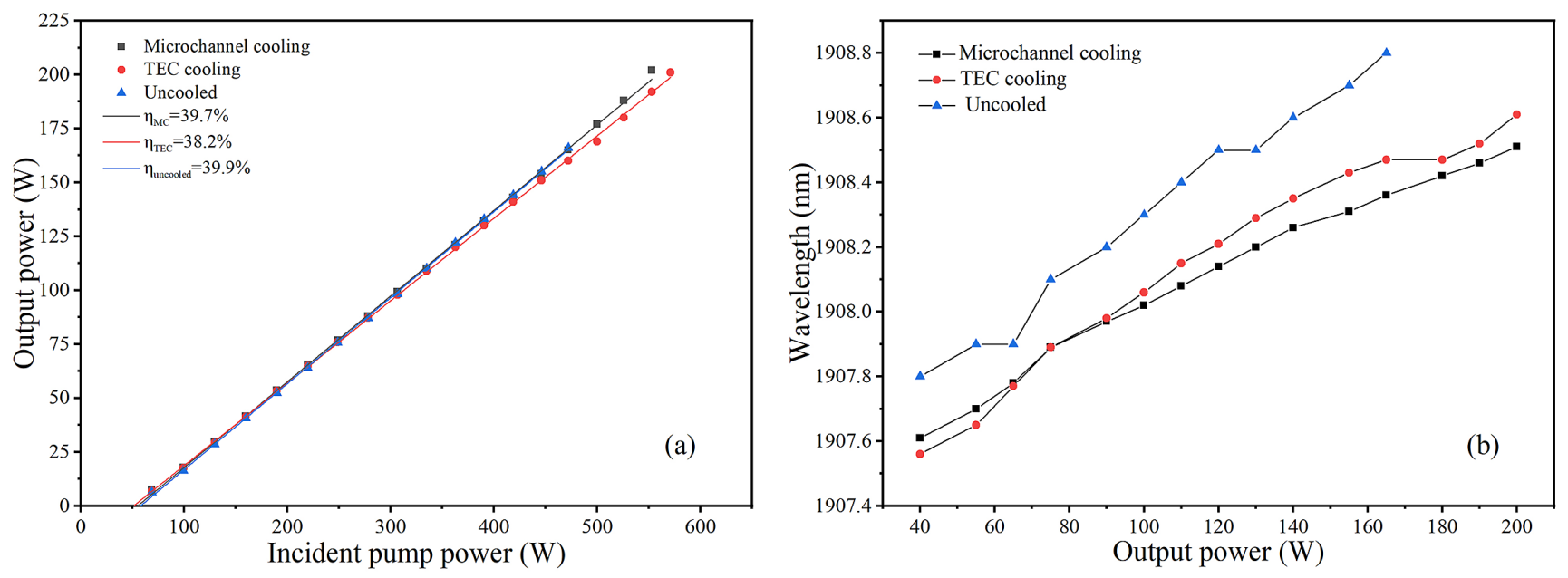

Figure 4. Comparison of CW laser performance, including (a) output power and (b) wavelength under different heat dissipation methods for the VBG.

in the two cases: less than $465 \mathrm{~W}$ without active cooling and $553 \mathrm{~W}$ under active cooling. Figure 4(b) shows that these methods successfully limit the growth rate of laser wavelength; especially the microchannel cooler is more effective. The corresponding wavelengths at 30 and $202 \mathrm{~W}$ were 1907.50 and $1908.51 \mathrm{~nm}$, respectively, leading to a shift of $1.01 \mathrm{~nm}$, which was much better than that without active cooling. Meanwhile, the TEC was also useful, but the effect was not as pronounced as that of microchannel cooler. The wavelength shifted by $1.1 \mathrm{~nm}$ from $1907.54 \mathrm{~nm}$ at $30 \mathrm{~W}$ to $1908.64 \mathrm{~nm}$ at $201 \mathrm{~W}$. In addition, the maximum temperature of the VBG was $389 \mathrm{~K}$ at $201 \mathrm{~W}$.

The 10/90 knife edge method was used to measure the spot radius at different positions, and the beam quality factor $M^{2}$ was further obtained by fitting the spot change curve. As shown in Figure 5, the beam quality factor $M^{2}$ was compared under different output power. When the $\mathrm{CW}$ output power was $160 \mathrm{~W}$ without active heat dissipation for the VBG, the beam quality factor in $x$ direction was $M^{2}{ }_{x}=2.5$, and the beam quality factor in $y$ direction was $M_{y}^{2}=4.0$, as shown in Figure 5(a); when the output power was $202 \mathrm{~W}$ with microchannel cooling, the beam quality factor in $x$ direction was $M_{x}^{2}=2.3$, and the beam quality factor in $y$ direction was $M^{2}{ }_{y}=4.0$, as shown in Figure 5(b). The results indicate that the beam quality of $202 \mathrm{~W}$ is equivalent to that of $160 \mathrm{~W}$, which largely depended on the use of active cooling to alleviate the deterioration of beam quality caused by the thermal distortion of the $\mathrm{VBG}^{[23]}$. Meanwhile, the beam analyzer (Pyrocam IV, OPHIR) was used to obtain the distribution of two-dimensional beam profiles at a distance of about $1500 \mathrm{~mm}$ from the coupler, as shown in Figure 5 (insets). The insets show that the beam profiles are similar in both cases, which are approximately ellipse. However, the beam quality of the laser built in this work has been greatly 

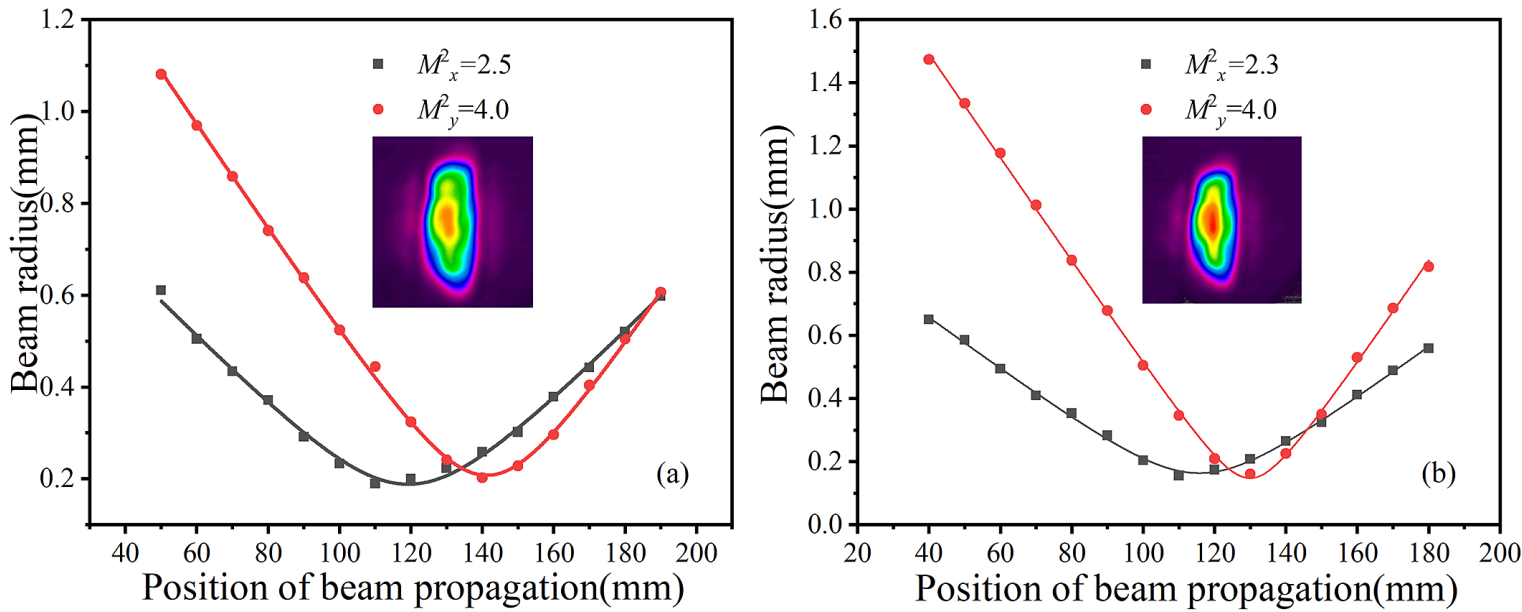

Figure 5. Beam quality at different average power levels: (a) beam quality of $160 \mathrm{~W}$ Tm:YLF laser without active cooling; (b) beam quality of $202 \mathrm{~W}$ Tm:YLF laser with microchannel cooling.

improved. Compared with the pump units reported in Refs. $[17,18]$, which consisted of multiple diode laser bars and generated pump lines $\left(0.53 \mathrm{~mm} \times 12 \mathrm{~mm}^{[17]}, 0.45 \mathrm{~mm} \times\right.$ $12 \mathrm{~mm}^{[18]}$ ) inside the crystal after passing through the imaging system, we used the $150 \mathrm{~W}$ fiber-coupled semiconductor laser and dual-end-pumped Tm:YLF slab with a uniform pump spot with a radius of $0.85 \mathrm{~mm}$, which was similar to the oscillating spot, achieving good mode matching.

In this paper, the thermal lensing effect in the Tm:YLF crystal is not negligible under high pump power ${ }^{[24,25]}$. As is well known, the crystal always operates as a divergent lens, resulting from the coupled effects of a negative $\mathrm{d} n / \mathrm{d} T$, and from side cooling. Then, the thermal lensing effect and oscillation mode at the highest output power of $202 \mathrm{~W}$ can be simply analyzed. The equivalent thermal focal length was deduced from the backward calculation with the measured $M^{2}$ factor ${ }^{[26]}$, which was about $-238 \mathrm{~mm}$ at $202 \mathrm{~W}$. According to the $A B C D$ matrix theory, the optical resonator met the stability criterion $|(A+D) / 2|<1$. The size of the oscillating spot at each crystal position can also be estimated. The radius of the resonator $\mathrm{TEM}_{00}$ mode within the length of the crystal (close to the mirror M3) was about from 0.92 to $1.0 \mathrm{~mm}$; and the radius in the other crystal (close to the VBG) was about from 0.65 to $0.7 \mathrm{~mm}$. Each pump light basically entered the crystal with a radius of $0.85 \mathrm{~mm}$, which basically satisfied the matching of high efficiency.

Microchannel cooling was used in the follow-up experiment. From Figure 3(a), the slope efficiency of the laser was $39.7 \%$, and the optical-to-optical conversion efficiency was $36.5 \%$. The central wavelength was $1908.5 \mathrm{~nm}$ recorded by a wavemeter (721 A, Bristol) and the linewidth (FWHM) was less than $0.6 \mathrm{~nm}$ at $202 \mathrm{~W}$, as shown in Figure 6. In addition, the laser was not damaged during the $40 \mathrm{~min}$ free operation of the laser at room temperature, and the power fluctuation was less than $1 \%$ of the maximum power.

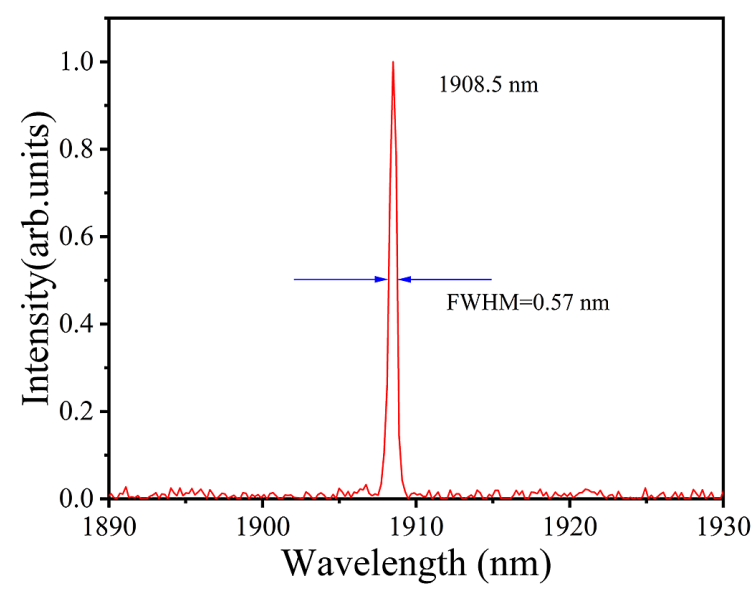

Figure 6. Spectrum of the Tm:YLF laser.

\section{Conclusion}

In summary, we have studied a $202 \mathrm{~W}$ two-crystal-in-series and dual-end-pumped Tm:YLF slab laser with a reflecting VBG as an output coupler. In order to suppress the growth of wavelength, the active heat dissipation methods were used for the VBG. The results show that the shift of wavelength was better suppressed by active heat dissipations; in particular, the effect of microchannel cooler was more prominent. When a microchannel cooler was used, the maximum CW output powers of $202 \mathrm{~W}$ at $1908.5 \mathrm{~nm}$ with linewidth (FWHM) $0.57 \mathrm{~nm}$ were obtained under an incident pump power of $553 \mathrm{~W}$, corresponding to a slope efficiency of $39.7 \%$ and optical-to-optical conversion efficiency of $36.5 \%$. We could also see the wavelength from $1907.5 \mathrm{~nm}$ at $30 \mathrm{~W}$ to $1908.5 \mathrm{~nm}$ at $202 \mathrm{~W}$ only shifted by $1.0 \mathrm{~nm}$, which was much better than that without active cooling. The beam quality factors $M^{2}$ were 2.3 and 4.0 for horizontal and vertical directions at $202 \mathrm{~W}$, respectively. 


\section{Acknowledgment}

This work was supported by the National Natural Science Foundation of China (No. U20A20214).

\section{References}

1. O. L. Antipov, N. G. Zakharov, M. Fedorov, N. M. Shakhova, N. N. Prodanets, L. B. Snopova, V. V. Sharkov, and R. Sroka, Med. Laser Appl. 26, 67 (2011).

2. T. J. Carrig, Proc. SPIE 5620, 187 (2004).

3. T. J. Wagener, N. Demma, J. D. Kmetec, and T. S. Kubo, in IEEE Digital Avionics Systems Conference (1995), p. 23.

4. G. Liu, S. Mi, K. Yang, D. Wei, J. Li, B. Yao, C. Yang, T. Dai, X. Duan, L. Tian, and Y. Ju, Opt. Lett. 46, 82 (2021).

5. H. Huang, S. Wang, H. Chen, O. L. Antipov, S. S. Balabanov, and D. Shen, Opt. Express 27, 38593 (2019).

6. A. Berrou, O. J. P. Collett, D. Morris, and M. J. D. Esser, Opt. Express 26, 10559 (2018).

7. P. A. Budni, M. L. Lemons, J. R. Mosto, and E. P. Chicklis, IEEE J. Sel. Top. Quantum Electron. 6, 629 (2000).

8. X. M. Duan, Y. X. Cai, Y. Ding, T. Y. Dai, and K. Zhao, Opt. Quantum Electron. 46, 1589 (2014).

9. B. M. Walsh, N. P. Barnes, M. Petros, J. Yu, and U. N. Singh, J. Appl. Phys. 95, 3255 (2004).

10. Y. Li, X.-Y. Chen, J.-L. Liu, C.-T. Wu, H. Dong, and G.-Y. Jin, J. Russ. Laser Res. 38, 470 (2017).

11. I. E. Gordon, L. S. Rothman, C. Hill, R. V. Kochanov, Y. Tan, P. F. Bernath, M. Birk, V. Boudon, A. Campargue, K. V. Chance, B. J. Drouin, J. M. Flaud, R. R. Gamache, J. T. Hodges, D. Jacquemart, V. I. Perevalov, A. Perrin, K. P. Shine, M. A. H. Smith, J. Tennyson, G. C. Toon, H. Tran, V. G. Tyuterev, A. Barbe, A. G. Császár, V. M. Devi, T. Furtenbacher, J. J. Harrison, J. M. Hartmann, A.
Jolly, T. J. Johnson, T. Karman, I. Kleiner, A. A. Kyuberis, J. Loos, O. M. Lyulin, S. T. Massie, S. N. Mikhailenko, N. Moazzen-Ahmadi, H. S. P. Müller, O. V. Naumenko, A. V. Nikitin, O. L. Polyansky, M. Rey, M. Rotger, S. W. Sharpe, K. Sung, E. Starikova, S. A. Tashkun, J. V. Auwera, G. Wagner, J. Wilzewski, P. Wcisło, S. Yu, and E. J. Zak, J. Quant. Spectrosc. Radiat. Transfer 203, 3 (2017).

12. A. Dergachev, P. F. Moulton, V. Smirnov, and L. Glebov, in Conference on Lasers and Electro-Optics (2004), p. 2.

13. T. H. Wang, Y. L. Ju, X. M. Duan, B. Q. Yao, X. T. Yang, and Y. Z. Wang, Laser Phys. Lett. 6, 117 (2009).

14. H. J. Strauss, M. J. D. Esser, G. King, and L. Maweza, Opt. Mater. Express 2, 1165 (2012).

15. T. Waritanant and T. Y. Chung, IEEE J. Quantum Electron. 47, 390 (2011).

16. S. Tjornhammar, B. Jacobsson, V. Pasiskevicius, and F. Laurell, J. Opt. Soc. Am. B 30, 1402 (2013).

17. J. Li, S. H. Yang, A. Meissner, M. Hoefer, and D. Hoffmann, Laser Phys. Lett. 10, 5 (2013).

18. Y. F. Mao and L. Wang, Laser Phys. 29, 115004 (2019).

19. O. M. Efimov, L. B. Glebov, V. I. Smirnov, and L. Glebova, US Patent: US 6586141 (2003).

20. X. Zhang, J. S. Feng, B. X. Xiong, K. S. Zou, and X. Yuan, Opt. Express 22, 8291 (2014).

21. M. S. Zediker, G. B. Venus, A. Sevian, V. I. Smirnov, and L. B. Glebov, Proc. SPIE 5711, 166 (2005).

22. P. Jelger, P. Wang, J. K. Sahu, F. Laurell, and W. A. Clarkson, Opt. Express 16, 9507 (2008).

23. X. Zheng, G. He, B. Zhang, J. Guo, Z. Jiao, and B. Wang, IEEE Photonics Technol. Lett. 28, 1107 (2016).

24. A. Jolly, S. Vidal, and J. Boullet, Laser Phys. 28, 065802 (2018).

25. X. Chen, J. Liu, C. Wu, R. Wang, and G. Jin, Integr. Ferroelectr. 210, 197 (2020).

26. B. Neuenschwander, R. Weber, and H. P. Weber, IEEE J. Quantum Electron. 31, 1082 (1995). 